\title{
Resultados Adversos do Parto em Maternidades de Ensino de Alto Risco
}

\section{Adverse Outcomes of Childbirth in High-Risk Maternity Hospitals}

\section{RESUMO}

Objetivo: Analisar os resultados adversos em maternidades de ensino de Maceió, Alagoas, Brasil. Metodologia: Estudo transversal, retrospectivo e analítico, com amostra aleatória de 480 prontuários de puérperas de parto cirúrgico de 2016, utilizando-se o Adverse Outcome Index: morte materna intrahospitalar, morte neonatal intra-hospitalar $\geq 2500 \mathrm{~g} \mathrm{e} \geq 37$ semanas, rotura uterina, admissão materna não planejada em unidade de terapia intensiva, trauma de parto no recémnascido, retorno à sala cirúrgica, admissão em unidade intensiva neonatal com $\geq 2500 \mathrm{~g}$ e $\geq 37$ semanas por mais de um dia, Apgar $<7$ no quinto minuto, hemotransfusão materna e laceração perineal de $4^{\circ}$ grau. Os dados foram analisados por meio do software Statistical Package for the Social Sciences versão 22.0. Resultados: A taxa de resultados adversos foi $21 \%$, na proporção de 26,4 para cada 1000 partos-dia, com maior ocorrência de admissão neonatal em terapia intensiva com $\geq 2500 \mathrm{~g}$ e $\geq 37$ semanas por mais de um dia $(52,5 \%)$, hemotransfusão materna $(20,8 \%)$ e admissão materna não planejada em terapia intensiva (17,8\%). Conclusão: Evidenciouse uma elevada parcela de nascimentos com resultados não desejáveis, o que proporcionou a análise do panorama dos desfechos desfavoráveis relacionados à segurança em maternidades através do uso de indicadores.

\section{DESCRITORES}

Avaliação de Resultados. Indicadores de Qualidade em Assistência à Saúde. Segurança do Paciente. Cuidado Perinatal. Maternidades.

\begin{abstract}
Objective: To analyze adverse outcomes in maternity teaching hospitals in Maceió, Alagoas, Brazil. Methodology: A crosssectional, retrospective and analytical study was carried out with a random sample of 480 medical records of postpartum women in 2016 using the Adverse Outcome Index: in-hospital maternal death, neonatal in-hospital death $>2500 \mathrm{~g}$ and $>37$ weeks, uterine rupture, unplanned maternal admission to intensive care unit, delivery trauma to the newborn, return to the operating room, admission to an intensive neonatal unit with $>2500$ g and $>37$ weeks for more than one day, Apgar $<7$ at the fifth minute, maternal hemotransfusion and fourth-degree perineal laceration. The data were analyzed using the Statistical Package for Social Sciences software version 22.0. Results: The results showed a $21 \%$ rate of adverse outcomes, at a rate of 26.4 for every 1000 births, with a greater occurrence of neonatal intensive care admission with $>2500 \mathrm{~g}$ and $>37$ weeks for more than one day $(52.5 \%)$, maternal blood transfusion $(20.8 \%)$ and unplanned maternal admission in intensive care (17.8\%). Conclusion: The evaluation of adverse outcomes evidenced a high proportion of births with undesirable results, which allowed the analysis of the outlook of unfavorable outcomes related to safety in maternity hospitals through the use of indicators.
\end{abstract}

\section{DESCRIPTORS}

Outcome Assessment. Quality Indicators. Health Care. Patient Safety. Perinatal Care. Maternity.

${ }^{1}$ Enfermeira, Mestre pelo Programa de Pós-Graduação da Escola de Enfermagem e Farmácia - ESENFAR da Universidade Federal de Alagoas - UFAL.

${ }^{2}$ Enfermeira, Professora Doutora da Escola de Enfermagem e Farmácia - ESENFAR da Universidade Federal de Alagoas - UFAL. 
gestação e o parto são eventos
fisiológicos que representam a
terceira causa de internação no Brasil, com graus de complexidade variados, desde condições naturais, na ausência de complicações, até a presença de patologias e situações que requerem demandas especializadas dos serviços de saúde ${ }^{1,2}$.

Os serviços de saúde na área obstétrica são pontos críticos para a qualidade do serviço hospitalar e a segurança do paciente ${ }^{3}$. Estimase que $29 \%$ das internações para o parto apresentem algum tipo de complicação ${ }^{4,5}$, com os danos relacionados à assistência (eventos adversos) correspondendo a 5 a $26 \%$ dos nascimentos ${ }^{4,5}$.

Além de incidentes comuns a todos os serviços de saúde, como quedas, eventos relacionados a medicamentos e hemocomponentes, existe uma gama de danos característicos da assistência perinatal: morte materna, eclâmpsia, rotura uterina, lacerações perineais e fístulas vaginais, dentre outros, que trazem elevado impacto social, econômico, ambiental, desconforto significativo na vida sexual e reprodutiva das mulheres ${ }^{6-8}$. Estes incidentes são relacionados à danos diretos da assistência ou omissão de condutas recomendadas na gestação e no parto ${ }^{8}$.

Os desfechos desfavoráveis ou complicações do parto podem ser utilizados na avaliação da qualidade do cuidado, por meio de indicadores do perfil de danos, o que proporciona a análise de causas, os fatores de risco e os resultados assistenciais ${ }^{9,10}$. A avaliação de resultados em serviços de saúde mostra-se vantajosa por associar medidas de desfechos com a avaliação da segurança do paciente ${ }^{11}$, representando incidentes e situações adversas que impactam nos sistemas de saúde.

O "Adverse Outcome Index" (AOI) do National Perinatal Information Center (NPIC) reflete um panorama dos resultados adversos no cuidado perinatal. $\mathrm{O}$ AOI contempla 10 indicadores: morte materna intra-hospitalar, morte neonatal intra-hospitalar $\geq 2500 \mathrm{~g}$ e $\geq 37$ semanas, rotura uterina, admissão materna não planejada em unidade de terapia intensiva, trauma de parto no RN, retorno à sala de parto/cirúrgica, admissão em unidade intensiva neonatal com $\geq 2500 \mathrm{~g}$ e $\geq 37$ semanas por mais de um dia, Apgar $<7$ no quinto minuto, hemotransfusão materna e laceração perineal de $4^{\circ}$ grau $^{3}$.

A análise de resultados adversos em maternidades de ensino de Alagoas, Brasil representou resultados do cuidado ao binômio e incorpora estratégias de avaliação significativas aos processos de prevenção, redução de riscos e danos decorrentes da assistência ${ }^{2,11}$. A construção de processos de gestão e avaliação pautados em resultados assistenciais que representam situações de risco deve ser incentivada em instituições de ensino como ferramenta de inclusão da cultura de segurança ao processo ensinoaprendizagem na área de saúde.

Considerando-se a grande demanda assistencial envolvida no cuidado perinatal, a abrangência dos resultados indesejáveis para esta clientela e a associação destes eventos com o processo biopsicossocial que abrange o parto e o nascimento ${ }^{3,12}$, o objetivo deste estudo foi analisar o índice de resultados adversos em maternidades de ensino de alto risco de Maceió, Alagoas, Brasil. 


\section{METODOLOGIA}

Estudo transversal, retrospectivo e analítico, com a utilização de dados secundários de registro de prontuários realizado em duas maternidades de ensino de Maceió, Alagoas, Brasil. As maternidades são vinculadas a duas universidades públicas de Alagoas, com diversos cursos que utilizam dos cenários das maternidades para práticas supervisionadas, estágios, programas de residência médica, de enfermagem e multiprofissional, além de projetos de extensão e pesquisa.

A clientela de alto risco é diversificada e contempla condições maternas e neonatais que requerem estrutura especializada para resolução de possíveis complicações, além de leitos de retaguarda de terapia intensiva adulto e neonatal, com equipamentos e recursos físicos específicos. Este perfil de público foi escolhido pela estreita relação com a morbimortalidade materna e infantil ${ }^{12}$.

A amostra foi composta por 480 prontuários e considerou como referência de cálculo as diretrizes propostas pelo instrumento adaptado do Institute for Healthcare Improvement (IHI) para avaliação de eventos adversos no parto ${ }^{13,14}$, avaliandose o ano de 2016, com a análise de 10 prontuários referentes a cada quinzena de 2016. A técnica de amostragem foi aleatória simples com a ferramenta de sorteio disponível no endereço: http://www.randomizer.org/form. $\mathrm{htm}$, para fornecer igual possibilidade dos prontuários serem incluídos no estudo.

Os critérios de inclusão foram: mulheres que tenham evoluído para desfecho de parto cirúrgico, com idade gestacional acima de 20 semanas, pela data da última menstruação (DUM ou ultrassonografia precoce), que permaneceram no mínimo 24 horas internadas em qualquer setor das maternidades; e que tiveram alta do serviço, independente do desfecho e seus prontuários foram fechados e encaminhados ao Serviço de Arquivo Médico. Foram excluídas pacientes que tiveram partos em outros serviços e que foram encaminhadas às maternidades do estudo, posteriormente.

Os indicadores do AOI foram relacionados às variáveis de admissão (perfil sociodemográfico e de internamento), desfechos do parto e características das cesáreas. Foram obtidos os indicadores de escores de gravidade - escore ponderado de resultados adversos (pontuação de gravidade segundo total de partos) e índice de gravidade (pontuação de gravidade segundo partos com resultados adversos) ${ }^{15}$.

Os dados foram armazenados em planilha do Microsoft Office Excel 2013 e para análise estatística foi utilizado o software IBM Statistical Package for the Social Sciences (SPSS) versão 22.0, ambos para Windows. Os dados descritivos foram operacionalizados por meio de tabelas, médias, frequências, desvio padrão e estatística inferencial com testes quiquadrado e exato de Fisher para variáveis qualitativas. O nível de significância estatística considerado foi $p<0,05$, a um intervalo de confiança estabelecido de $95 \%$.

A aprovação no Comitê de Ética foi estabelecida pelo o parecer CAAE 61093616.8.0000.5013 e foi obtida anuência dos gestores dos hospitais. O estudo seguiu normas da Resolução No 466 de 2012, do Conselho Nacional de Saúde do Brasil. 


\section{RESULTADOS}

Em 2016, ocorreram 1958 cesáreas nos hospitais analisados. Foram revisados 480 ( $24,5 \%$ de todas as cesáreas) prontuários de mulheres submetidas a parto cirúrgico, o que representou 3812 partos-dia (total de dias de internamento para o parto). A idade variou de 13 a 46 anos, com uma média de $25 \pm 7,3$ anos, sendo $16,2 \%$ com idade inferior a 18 anos; 58,6\% das mulheres tinham escolaridade inferior a nove anos de estudo, 56,3\% exerciam alguma atividade remunerada, 97,3\% eram de etnia parda, $52,4 \%$ possuíam companheiro e $52,3 \%$ provinham de outros municípios do interior do estado.

$\mathrm{Na}$ admissão, $73,5 \%$ tinham acompanhante, $37,3 \%$ eram primigestas, $33,3 \%$ tiveram cesáreas anteriores e $26,6 \%$ tiveram abortamentos. A idade gestacional na admissão foi de 20 a 42 semanas, com média de 36 semanas e 05 $\pm 3,2$ dias, sendo $51,8 \%$ admitidas antes de 37 semanas. O tempo de permanência médio foi de $08 \pm 7,6$ dias. $\mathrm{Na}$ admissão, apenas 10,4\% estavam em trabalho de parto e a apresentação cefálica foi relatada em $36,3 \%$ das admissões, apesar da informação estar ausente em $60 \%$ dos registros. $O$ relato de perda líquida e da rotura de membranas esteve presente em $24,9 \%$ dos prontuários. A pressão arterial na admissão mostrou-se elevada, sendo as médias da sistólica $137 \mathrm{mmHg} \pm 23 \mathrm{mmHg}$ e a diastólica $87 \mathrm{mmHg} \pm 17 \mathrm{mmHg}$.

As causas de internação se assemelharam ao perfil de indicações de cesárea, com $38,1 \%$ relacionadas às desordens hipertensivas, $18,3 \%$ a intercorrências no parto e $15,2 \%$ ao sofrimento fetal agudo. As cesáreas ocorreram quando as mulheres estavam em trabalho de parto em $13,8 \%$ dos casos. Um quarto dos procedimentos foi de urgência e o tipo de anestesia em $96,5 \%$ foi o bloqueio neuroaxial. Após o parto, $4,2 \%$ das puérperas foram encaminhadas à UTI e 36,9\% dos RN necessitaram de cuidados neonatais específicos, com média de peso ao nascer de $2984 \pm 869 \mathrm{~g}$. Ocorreram 12 óbitos fetais na amostra pesquisada.

A frequência de resultados adversos foi de $21 \%$, sendo $26,4 / 1000$ partos-dia, o escore ponderado de resultados adversos de 8,11 e índice de gravidade de 38,56. A Tabela 1 descreve a frequência dos resultados adversos e o índice de gravidade.

Os indicadores mais frequentes foram admissão neonatal em UTI em 11\%, hemotransfusão materna em 4,3\% e admissão materna não planejada em UTI em 3,7\%. Os indicadores de morte materna, morte neonatal intra-hospitalar de RN >2500g/>37semanas e laceração perineal de quarto grau não foram encontrados.

O perfil de atendimento foi característico de serviços de alto risco. As mulheres admitidas com história de gestações anteriores (multigestas), encaminhadas via regulação de leitos de outra instituição, admitidas antes de 37 semanas e que tiveram acompanhante no momento do internamento evoluíram com mais frequência para resultados adversos.

Os valores de pressão arterial $<120 / 80 \mathrm{mmHg}$ foram associados a maior ocorrência do desfecho, muito embora o aumento motive elevada parcela 
Tabela 1. Indicadores de resultados adversos e escores de gravidade* em maternidades de ensino de Maceió, AL, Brasil, 2016.

\begin{tabular}{|c|c|c|c|c|}
\hline \multirow[t]{2}{*}{ Indicador } & \multirow[t]{2}{*}{$\mathrm{n}$} & \multirow[t]{2}{*}{$\%$} & \multicolumn{2}{|c|}{ Escores de gravidade } \\
\hline & & & Pontos & Total \\
\hline Morte materna intra-hospitalar & - & - & 750 & - \\
\hline $\begin{array}{l}\text { Óbito neonatal intra-hospitalar em } \geq 2500 \text { gramas } \\
\text { ou } \geq 37 \text { semanas }\end{array}$ & - & - & 400 & - \\
\hline Rotura uterina no trabalho de parto & 02 & 02 & 100 & 200 \\
\hline Admissão materna não planejada em UTI† & 18 & 17,8 & 65 & 1170 \\
\hline Trauma de parto & - & - & 60 & - \\
\hline Retorno antecipado à sala cirúrgica & 5 & 4,9 & 40 & 200 \\
\hline $\begin{array}{l}\text { Admissão em UTI† neonatal de RN } ¥ \geq 2500 \\
\text { gramas ou } \geq 37 \text { semanas por }>1 \text { dia }\end{array}$ & 53 & 52,5 & 35 & 1855 \\
\hline Apgar $<7$ no $5^{\circ}$ minuto & 02 & 02 & 25 & 50 \\
\hline Hemotransfusão maternal & 21 & 20,8 & 20 & 420 \\
\hline Laceração perineal de $4^{\circ}$ grau & - & - & 05 & - \\
\hline Total & 101 & 100 & - & 3895 \\
\hline
\end{tabular}

Nota: *Segundo Mann et al. (2006); †UTI - unidade de terapia intensive; †RN - recém-nascido.

de internamento em serviços de maior complexidade e tenha sido responsável por $53,5 \%$ das causas de internamento relacionadas à ocorrência de resultados adversos.

As mulheres com idade entre 19 e 34 anos, que exerciam atividade remunerada, internadas por causas hipertensivas e cujos RN tiveram baixo peso ao nascer apresentaram maior ocorrência de admissão em UTI neonatal. As mulheres pardas apresentaram mais retornos à sala cirúrgica e aquelas encaminhadas à enfermaria após o parto necessitaram com mais frequência de hemotransfusão. Os fatores que influenciaram a admissão materna não planejada em UTI foram admissão e parto antes de 37 semanas, presença do acompanhante, pressão arterial elevada na admissão, parto de urgência/ emergência e peso do RN. As Tabelas 2 e 3 detalham a relação entre os indicadores mais frequentes e as variáveis sociodemográficas, de acesso ao serviço e de desfechos do parto.

\section{DISCUSSÃO}

A frequência de $\mathrm{AOI}$ deste estudo (21\%) mostrou-se elevada comparando-se a outros autores ${ }^{11,15}$. Este índice mostrou-se mais frequente entre mulheres pardas, procedentes do interior, da faixa etária adulta-jovem (19-34 anos), com menor escolaridade (menos de nove anos de estudo), sem companheiro e que exercem atividade remunerada.

Tais associações entre os desfechos desfavoráveis e o perfil sociodemográfico são resultados da influência direta no acesso aos serviços de saúde e nas questões de renda, o que se reflete no perfil de morbimortalidade materna e infantil de forma geral. Em outro estudo, gestantes não brancas, casadas, com escolaridade até $2^{\circ}$ grau incompleto, procedentes do interior e com renda familiar de até um salário mínimo estiveram sujeitas a maior risco de complicações potencialmente letais ${ }^{16}$.

O modelo obstétrico contemporâneo, principalmente no Brasil, expõe as mulheres 
Tabela 2. Distribuição dos resultados adversos segundo perfil da amostra em maternidades de ensino, Maceió, AL, Brasil, 2016.

\begin{tabular}{|c|c|c|c|c|c|c|c|c|c|c|c|c|}
\hline \multirow[t]{2}{*}{ Variáveis } & \multicolumn{3}{|c|}{$\begin{array}{c}\text { Hemotransfusão } \\
\text { materna }\end{array}$} & \multicolumn{3}{|c|}{$\begin{array}{c}\text { Admissão } \\
\text { materna em UTI* }\end{array}$} & \multicolumn{3}{|c|}{$\begin{array}{l}\text { Retorno à sala } \\
\text { cirúrgica }\end{array}$} & \multicolumn{3}{|c|}{$\begin{array}{c}\text { Admissão } \\
\text { neonatal em UTI* }\end{array}$} \\
\hline & $\mathrm{n}$ & $\%$ & $p^{*}$ & $\mathrm{n}$ & $\%$ & $p^{*}$ & $\mathrm{n}$ & $\%$ & $p^{*}$ & $\mathrm{n}$ & $\%$ & $\mathrm{p}^{*}$ \\
\hline $\begin{array}{l}\text { Faixa etária } \\
\leq 18 \\
19 \text { a } 34 \\
\geq 35\end{array}$ & $\begin{array}{l}05 \\
15 \\
01\end{array}$ & $\begin{array}{c}23,8 \\
71,4 \\
4,7\end{array}$ & 0,298 & $\begin{array}{l}01 \\
12 \\
05\end{array}$ & $\begin{array}{l}05,5 \\
66,7 \\
27,8\end{array}$ & 0,404 & $\begin{array}{l}- \\
04 \\
01\end{array}$ & $\begin{array}{l}- \\
80 \\
20\end{array}$ & 0,609 & $\begin{array}{l}03 \\
32 \\
18\end{array}$ & $\begin{array}{l}05,7 \\
60,3 \\
34,0\end{array}$ & $<0,001$ \\
\hline $\begin{array}{l}\text { Estudo (Anos) } \\
<9 \\
\geq 9\end{array}$ & $\begin{array}{l}08 \\
07\end{array}$ & $\begin{array}{l}53,3 \\
46,7\end{array}$ & 0,676 & $\begin{array}{l}09 \\
03\end{array}$ & $\begin{array}{l}75 \\
25\end{array}$ & $0,373 \ddagger$ & $\begin{array}{c}05 \\
-\end{array}$ & $\begin{array}{c}100 \\
-\end{array}$ & $0,79 \ddagger$ & $\begin{array}{l}23 \\
15\end{array}$ & $\begin{array}{l}60,5 \\
39,5\end{array}$ & 0,794 \\
\hline $\begin{array}{l}\text { Trabalho } \\
\text { Sim } \\
\text { Não }\end{array}$ & $\begin{array}{l}14 \\
07\end{array}$ & $\begin{array}{l}66,7 \\
33,3\end{array}$ & 0,329 & $\begin{array}{l}11 \\
07\end{array}$ & $\begin{array}{l}61,1 \\
38,9\end{array}$ & 0,677 & $\begin{array}{l}02 \\
02\end{array}$ & $\begin{array}{l}50 \\
50\end{array}$ & $01 \ddagger$ & $\begin{array}{l}38 \\
14\end{array}$ & $\begin{array}{l}73 \\
27\end{array}$ & 0,01 \\
\hline $\begin{array}{l}\text { Etnia } \\
\text { Branca } \\
\text { Parda } \\
\text { Preta }\end{array}$ & $\begin{array}{l}01 \\
17 \\
-\end{array}$ & $\begin{array}{c}5,5 \\
94,4 \\
-\end{array}$ & 0,087 & $\begin{array}{l}- \\
15 \\
-\end{array}$ & $\begin{array}{c}- \\
100 \\
-\end{array}$ & 0,807 & $\begin{array}{l}01 \\
04 \\
-\end{array}$ & $\begin{array}{c}20 \\
80 \\
-\end{array}$ & $<0,001$ & $\begin{array}{c}- \\
46 \\
01\end{array}$ & $\begin{array}{c}- \\
97,9 \\
2,1\end{array}$ & 0,777 \\
\hline $\begin{array}{l}\text { Logradouro } \\
\text { Maceió } \\
\text { Outro }\end{array}$ & $\begin{array}{l}09 \\
12\end{array}$ & $\begin{array}{l}42,9 \\
57,1\end{array}$ & 0,649 & $\begin{array}{l}06 \\
12\end{array}$ & $\begin{array}{l}33,3 \\
66,7\end{array}$ & 0,213 & $\begin{array}{l}02 \\
03\end{array}$ & $\begin{array}{l}40 \\
60\end{array}$ & $01 \ddagger$ & $\begin{array}{l}25 \\
28\end{array}$ & $\begin{array}{l}47,1 \\
52,8\end{array}$ & 0,934 \\
\hline $\begin{array}{l}\text { Estado civil } \\
\text { Companheiro } \\
\text { Sem } \\
\text { companheiro }\end{array}$ & $\begin{array}{l}06 \\
13\end{array}$ & $\begin{array}{l}31,6 \\
68,4\end{array}$ & 0,063 & $\begin{array}{l}11 \\
05\end{array}$ & $\begin{array}{l}68,7 \\
31,3\end{array}$ & $0,211 \ddagger$ & $\begin{array}{l}03 \\
02\end{array}$ & $\begin{array}{l}60 \\
40\end{array}$ & $01 \ddagger$ & 24 & $\begin{array}{l}47 \\
53\end{array}$ & 0,412 \\
\hline $\begin{array}{l}\text { Pré-natal } \\
\text { Sim } \\
\text { Não }\end{array}$ & $\begin{array}{c}08 \\
-\end{array}$ & $\begin{array}{c}100 \\
-\end{array}$ & 01 & $\begin{array}{c}08 \\
-\end{array}$ & $\begin{array}{c}100 \\
-\end{array}$ & $01 \ddagger$ & $\begin{array}{c}03 \\
-\end{array}$ & $\begin{array}{c}100 \\
-\end{array}$ & $01 \ddagger$ & $\begin{array}{c}23 \\
-\end{array}$ & $\begin{array}{c}100 \\
-\end{array}$ & $01 \ddagger$ \\
\hline $\begin{array}{l}\text { História } \\
\text { obstétrica } \\
\text { Primigesta } \\
\text { Multigesta }\end{array}$ & $\begin{array}{l}09 \\
12\end{array}$ & $\begin{array}{l}42,9 \\
57,1\end{array}$ & 0,611 & $\begin{array}{l}05 \\
13\end{array}$ & $\begin{array}{l}27,2 \\
72,2\end{array}$ & $0,463 \ddagger$ & $\begin{array}{l}03 \\
02\end{array}$ & $\begin{array}{l}60 \\
40\end{array}$ & $0,369 \ddagger$ & $\begin{array}{l}17 \\
36\end{array}$ & $\begin{array}{l}31 \\
69\end{array}$ & 0,378 \\
\hline $\begin{array}{l}\text { Idade } \\
\text { gestacional na } \\
\text { admissão } \\
<37 \text { semanas }\end{array}$ & 15 & 71,4 & 0,065 & 15 & 88,2 & $0,002 \ddagger$ & 03 & 60 & $01 \ddagger$ & 27 & 51 & 01 \\
\hline$\geq 37$ semanas & 06 & 28,6 & & 02 & 11,8 & & 02 & 40 & & 26 & 49 & \\
\hline $\begin{array}{l}\text { Acompanhante } \\
\text { Sim } \\
\text { Não }\end{array}$ & $\begin{array}{l}09 \\
02\end{array}$ & $\begin{array}{l}81,9 \\
18,1\end{array}$ & $0,160 \dagger$ & $\begin{array}{l}08 \\
03\end{array}$ & $\begin{array}{l}72,7 \\
27,3\end{array}$ & $0,03 \ddagger$ & $\begin{array}{c}04 \\
-\end{array}$ & $\begin{array}{c}100 \\
-\end{array}$ & 01‡ & $\begin{array}{l}34 \\
05\end{array}$ & $\begin{array}{l}87,1 \\
12,9\end{array}$ & $0,161 \ddagger$ \\
\hline
\end{tabular}

Nota: ${ }^{\star} T$ Teste quiquadrado; $¥$ Teste exato de Fisher.

e os recém-nascidos a altas taxas de intervenções com grande potencial de provocar danos. Tais intervenções, como a episiotomia, o uso de ocitocina e a cirurgia são rotineiras e atingem quase a totalidade das mulheres nas maternidades. Mesmo nas situações onde as complicações já existam, uma assistência inadequada não é capaz de reduzir os agravos delas resultantes, além de potencializá-los resultando, muitas vezes, na morte evitável de mulheres e crianças ou em lesões e sequelas permanentes ${ }^{17}$. 
Tabela 3. Distribuição dos resultados adversos segundo características do atendimento e cesáreas em maternidades de ensino, Maceió, AL, Brasil, 2016.

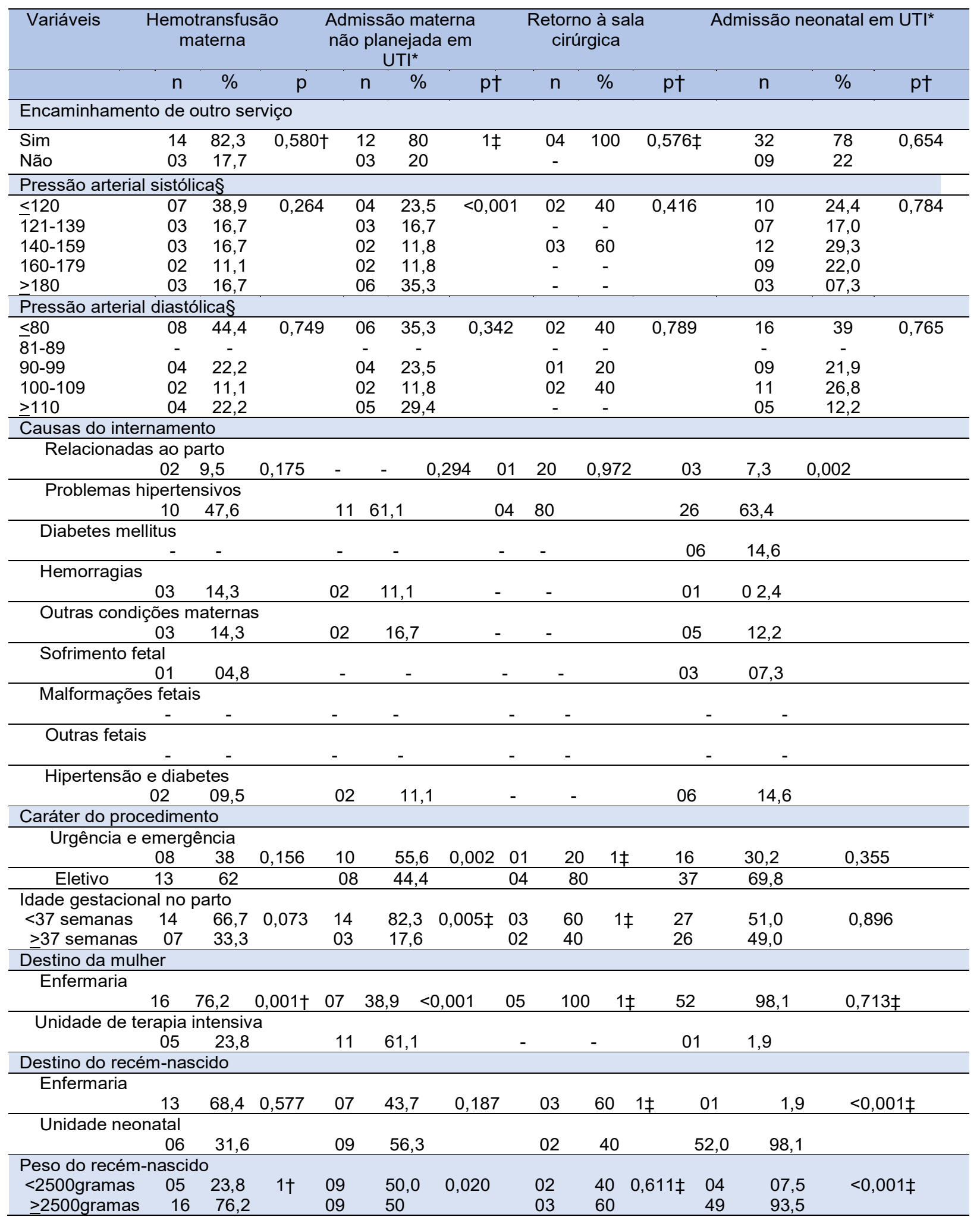

Nota: *UTI - unidade de terapia intensiva; †Teste quiquadrado; $¥$ Teste exato de Fisher; §Em milímetros de mercúrio. 
Os resultados adversos podem refletir a ocorrência de EA ao serem analisados do ponto de vista de resultados assistenciais e danos não intencionais decorrentes de problemas relacionados ao cuidado e seguimento clínico da gestação e do parto. A avaliação dos EA do ponto de vista global por meio do rastreamento de indicadores em registros de prontuários pode sinalizar intercorrências ou danos que em sua maioria são evitáveis na assistência ao parto, mas representam grande repercussão na saúde da mulher e do neonato ${ }^{18-21}$.

As mortalidades materna e neonatal são consideradas eventos sentinela e indicadores indiretos das condições de vida e de saúde de um país ${ }^{22}$. Enquanto, nos indicadores de desfecho final existe uma tendência de redução crescente, mas, apesar de pouco frequente, a morte materna apresenta-se com um alto valor preditivo para a ocorrência de danos durante a assistência ${ }^{20}$. Não ocorreram relatos de morte materna ou neonatal intra-hospitalar ( $\geq 37$ semanas e $\geq 2500 \mathrm{~g}$ ) na amostra analisada, fato possivelmente associado ao perfil de alta complexidade dos serviços analisados, ao acesso à tecnologias e recursos para resolução de situações potencialmente fatais.

As ocorrências não planejadas durante o internamento para o parto também podem estar relacionadas a resultados adversos. Neste estudo, a admissão materna não planejada em UTI, hemotransfusão materna e o retorno à sala de cirurgia representaram $43 \%$ dos resultados adversos e podem representar a tentativa de correção de algum incidente durante a assistência à mulher. Estes desfechos clínicos da mulher, assim como do RN tanto se relacionam ao nível e complexidade de cuidados envolvidos, quanto às dificuldades operacionais e assistenciais no manejo do parto e do nascimento.

Em hospitais belgas, foi estimado que um quarto das admissões em serviços mais complexos se deve a danos decorrentes da assistência ${ }^{23}$. O retorno à sala de cirurgia ou à sala de parto representou baixa especificidade, devido a relação com o motivo que levou ao retorno, que poderia ser decorrente de danos da assistência ou de necessidades específicas associadas a condições clínicas da mulher ${ }^{18,19}$.

A rotura uterina durante $o$ trabalho de parto, enquanto resultado adverso relacionase à rapidez, efetividade e segurança do cuidado à mulher; apesar de ser pouco frequente, associa-se a elevada morbidade e mortalidade, além de acarretar danos permanentes à vida reprodutiva da mulher ${ }^{21-24}$. Nos prontuários analisados, a rotura uterina esteve presente em $0,4 \%$ da amostra, o que representou $2 \%$ dos resultados adversos encontrados, indicador semelhante ao relatado por outros autore ${ }^{24} \mathrm{e}$ atuando como indicadorchave de complicações hemorrágicas mal conduzidas.

As condições de nascimento do RN podem indicar falhas no manejo da gestação e parto e, em se tratando de desfechos desfavoráveis, trazer consequências para o crescimento e o desenvolvimento da criança. $A$ admissão em UTI de neonatos de termo e com peso $\geq 2500 \mathrm{~g}$ foi o resultado mais frequente $(52,5 \%)$ e teve relação significativa com a faixa etária da mãe (19-34 anos) e a variável trabalho. Tal fato, pode ser justificado pelo tipo de parto analisado e pelas características da clientela, com situações de alto risco 
e demandas de maior complexidade, que podem aumentar o risco de admissão neonatal em UTI.

Junto à admissão neonatal em UTI, o Apgar < 7 no quinto minuto representa indicador com maior valor preditivo em comparação à presença de trauma de parto no RN, o qual esteve associado a erros de registro e dificuldades de identificação em outros estudos ${ }^{19} \mathrm{e}$, particularmente nesta pesquisa, foi um resultado sem nenhuma ocorrência.

Os danos na assistência perinatal abrangem diversas vertentes: erros decorrentes da assistência; intervenções desnecessárias e/ou inoportunas que comprometem a evolução do parto e proporcionam danos; omissões de condutas comprovadamente eficazes, o que implica resultados menos favoráveis, negativos ou não esperados para o seguimento clínico da gestação, do parto e do puerpério $^{25}$. O uso de conjuntos de indicadores para representar diferentes vertentes pode proporcionar vigilância de incidentes, eventos sentinela e resultados do cuidado.

\section{CONCLUSÃO}

O índice de resultados adversos na amostra foi de $21 \%$, sendo 26,4 por cada 1000 partos-dia, e o escore ponderado de 8,11 e 38,56 em relação ao índice de gravidade.
Mulheres da faixa etária adulta-jovem (1934 anos), pardas, sem companheiro, com atividade remunerada e de menor escolaridade tiveram maior ocorrência de resultados adversos.

Os valores de pressão arterial à admissão nos níveis de normalidade, presença de acompanhante, encaminhamento de outro serviço, história de gestações anteriores e admissão antes do termo se relacionaram à maior frequência de resultados adversos. As mulheres que evoluíram para cesáreas eletivas e que foram encaminhadas à enfermaria após o parto também tiveram maior expressão de resultados adversos.

Logo, os indicadores de resultados com maior representatividade foram admissão neonatal em UTI com $\geq 37$ semanas e/ ou $\geq 2500 \mathrm{~g}$, hemotransfusão materna e admissão materna não planejada em UTI. Os indicadores que não se apresentaram na amostra foram morte materna intra-hospitalar, morte neonatal intra-hospitalar $>37$ semanas e/ou $>2500 \mathrm{~g}$, trauma de parto e laceração perineal de $4^{\circ} \mathrm{grau}$.

As limitações do estudo se relacionaram ao fato da pesquisa ser documental retrospectiva, pela baixa qualidade dos registros em prontuários, o que contribui para a omissão de informações e a ausência de dados relevantes.

\section{REFERÊNCIAS}

1. Ministério da Saúde. Sistema de Informações Hospitalares do SUS (SIH/SUS). Internações Hospitalares do SUS por local de internação. [Internet]. 2016.

2. Suárez-Cortés $M$, Armero-Barranco $D$, Canteras-Jordana $\mathrm{M}$, Martínez-Roche ME. Use and influence of Delivery and Birth Plans in the humanizing delivery process. Rev. Latino-Am. Enfermagem. 2015; 23(3):520-6. 
3. Draycott $T$, Sagar R, Hogg $S$. The role of insurers in maternity safety. Best Pract Res Clin Obstet Gynaecol. 2015; 29(8):1126-31.

4. Berg CJ, Callaghan WM, Syverson C, Henderson Z. Pregnancy-related mortality in the United States, 19982005. Obstet Gynecol. 2010; 116(6):1302-1309.

5. Forster AJ, Fung I, Caughey S, Oppenheimer L, Beach C, Shojania KG, Van Walraven C. Adverse events detected by clinical surveillance on an obstetric service. Obstet Gynecol. 2006; 108(5):1073-1083.

6. Maia CS, Freitas DRC, Gallo LG, Araújo WN. Registry of adverse events related to health care that results in deaths in Brazil, 2014-2016. Epidemiol. Serv. Saude. 2018; 27(2):e2017320.

7. Aitken Z, Garrett CC, Hewitt B, Keogh L, Hocking JS, Kavanagh AM. The maternal health outcomes of paid maternity leave: a systematic review. Soc Sci Med. 2015; 130:32-41.

8. Kassebaum NJ, Bertozzi-Vila A, Coggeshall MS, Shackelford KA, Steiner C, Heuton KR, et al. Global, regional, and national levels and causes of maternal mortality during 1990-2013: a systematic analysis for the global burden of disease study 2013. Lancet. 2014; 384:980-1004.

9. Donabedian A. The quality of care. How can it be assessed? JAMA. 1988; 260(12):1743-8.

10. Lindsay P. Maternity support workers and safety in maternity care in England. Pract midwife. 2014; 17(10):2022.

11. Foglia EE, Nolen TL, DeMauro SB, Bell EF, Stoll BJ, Schmidt B. Short-term Outcomes of Infants Enrolled in Randomized Clinical Trials vs Those Eligible but Not Enrolled. JAMA. 2015; 313(23):2377-2379.

12. Goldenberg RL, Saleem S, Ali S, Moore JL, Lokangako A, Tshefu A, et al. Maternal near miss in low-resource areas. International Journal of Gynecology \& Obstetrics. 2017; 138(3):347-355.

13. Hibbert PD, Molloy CJ, Hooper TD, Wiles LK, Runciman WB, Lachman P, et al. The application of the Global Trigger Tool: a systematic review. Int J Qual Health Care. 2016; 28(6):640-649.

14. Tolcher MC, Torbenson VE, Weaver AL, McGree ME, El-Nashar SA, Nesbitt KM, et al. Impact of a labor and delivery safety bundle on a modified adverse outcomes index. Am J Obstet Gynecol. 2016; 214(3):401.

15. Mann S, Pratt S, Gluck P, P Nielsen, Risser D, Greenberg $P$, et al. Assessing quality obstetrical care: development of standardized measures. Jt Comm J Qual Patient Saf. 2006; 32(9):497-505.
16. Rosendo TMSS, Roncalli AG. Maternal near misses and health inequalities: an analysis of contextual determinants in the State of Rio Grande do Norte, Brazil. Ciênc. Saúde Coletiva. 2016; 21(1):191-201.

17. Fogaça VD, Schneck CA, Riesco MLG. Obstetric interventions during labor in women submitted to cesarean section. Cogitare Enferm. 2007; 12(3):296.

18. Howell EA, Zeitlin J, Hebert PL, Balbierz A, Egorova $\mathrm{N}$. Association between hospital-level obstetric quality indicators and maternal and neonatal morbidity. JAMA. 2014; 312(15):1531-1541.

19. Mhamdi ES, Ben SK, Bouanene I, Soussi SM Chronological observation of the epidemiological characteristics of perinatal indicators in the Monastir health region (Tunisia) between 1994 and 2008. Sante Publique. 2011; 23(4):287-295.

20. Atallah F, Bernstein PS, Diaz DA, Minkoff H. The Adverse Outcome Index: Putting Quality Into an Outcome Measure. Obstet Gynecol. 2018; 132(3):750-753.

21. Asgari H, Esfahani SS, Yaghoubi M, Javadi M, Karimi $\mathrm{S}$. Investigating selected patient safety indicators using medical records data. J Educ Health Promot. 2015; 6(4):54.

22. Clark SL, Meyers JA, Frye DR, McManus K, Perlin JB. A systematic approach to the identification and classification of near-miss events on labor and delivery in a large, national health care system. Am J Obstet Gynecol. 2012; 207(6):441-445

23. Marquet K, Claes N, De Troy E, Kox G, Droogmans M, Schrooten W, et al. One Fourth of Unplanned Transfers to a Higher Level of Care Are Associated With a Highly Preventable Adverse Event: A Patient Record Review in Six Belgian Hospitals. Critical Care Medicine. 2015; 43(5):1053-1061.

24. Pettker CM. Systematic approaches to adverse events in obstetrics, Part I: Event identification and classification. Seminars in Perinatology. 2017; 43(3):151-155.

25. Berg CJ, Callaghan WM, Syverson C, Henderson Z. Pregnancy-related mortality in the United States, 19982005. Obstet Gynecol. 2010; 116(6):1302-1309.

\section{CORRESPONDÊNCIA}

Thais da Costa Oliveira

Rua Doutor Leopoldo Florentino de

Oliveira e Silva, 12 A, Quadra J,

Residencial Monte Verde, Antares,

Maceió, Alagoas, CEP 57048040.

E-mail: enfthais.costa@outlook.com 